\title{
Broadening horizons
}

\author{
Acta Neuropsychiatrica 2006: 18:181-182. (C) Blackwell Munksgaard 2006
}

This October issue of Acta Neuropsychiatrica includes manuscripts that tackle a broad range of topics ranging from depression to substance misuse. A number of articles describe the pathophysiology of neuropsychiatric disorders and draw on neurobiological models from fields as diverse as endocrinology and neurocognition. Neuroimaging is a popular theme that features in three of the research articles. Yücel et al. (1), for instance, report intriguing structural magnetic resonance imaging findings in men with a history of both alcohol and cannabis use, noting significant changes in the size of key structures such as the amygdala and the hippocampus. This is an important report as it fuels the ongoing discussion that surrounds putative structural changes in drug use with respect to their significance. The possibility of a neurodevelopmental insult has only recently been mooted; however, evidence with respect to this remains inconsistent. Examining the other end of the age spectrum, a number of groups have posited late-onset changes, with the suggestion that this perhaps has a vascular basis. That lesions vascular or neoplastic can cause 'psychiatric symptoms' is well documented, and in keeping with this, Spengos et al. (2) in this issue describe a case of stroke-related peduncular hallucinosis, which underscores the importance of considering organic etiology and comorbidity.

Switching tack, Porter and Gallagher (3) distinguish themselves with a comprehensive review of hypothalamic-pituitary-adrenal (HPA) axis abnormalities in affective disorders. Gratifyingly, in addition to synthesizing a wealth of literature pertaining to the neuroendocrine pathophysiology of mood disorders (examined across both unipolar and bipolar subtypes, dealing with psychosis separately), they discuss in detail the treatments that can potentially modify HPA axis functioning, in particular mefipristone, a glucocorticoid receptor antagonist, and in doing so, impact on mood. Interestingly, the authors also comment on an apparent rapid response of patients with depression to antiglucocorticoid strategies, suggesting the emergence of a treatment response within 1 week, which is clinically significant by day $10-14$. This is salient clinically not only because of the potential immediate benefit this can confer but also because it hints at an alternate pathway to the core pathophysiology of depression. In support of this neuroendocrine axis changes can also impair cognition, as has been described previously by researchers within this group.

In an analysis that is a useful extension of a previous study, Goldney and Bain (4) describe the prevalence of 'double depression', namely the occurrence of major depression superimposed on dysthymia, across a representative community sample comprising both metropolitan and rural inhabitants. Specifically, they examine the use of services by this 'group' of patients and report that this is significantly higher than comparison patients with either dysthymia or major depression alone. Interestingly, they go on to show that both pharmacological and nonpharmacological treatments are not optimum with doses of antidepressant medications for instance below the maximum recommended doses and scant use of appropriate augmentation strategies. This is important as they point out because of the cost of depression and is most likely a consequence of a failure to translate research findings into community practice.

A brief overview of some of the articles in this issue highlights our intention to broaden the remit of this journal, with greater focus on emerging technologies and translational research. This is mirrored by the advisory board of Acta Neuropsychiatrica that has been further bolstered by the addition of editors from complementary journals so as to provide thematic breadth. The journal is now fully online with respect to submission and review, and currently, decisions on submitted 
manuscripts are made within 12 weeks from the time of submission.

\section{Gin S. Malhi}

\section{References}

1. Yücel M, Lubman DI, Velakoulis D et al. Structural brain correlates of alcohol and cannabis use in recreational users. Acta Neuropsych 2006;18:226-229.
2. Spengos K, Tsivgoulis G, Papadimitriou GN, Spengos M. Magnetic resonance imaging findings in a case of stroke-related peduncular hallucinosis. Acta Neuropsych 2006;18:230-231.

3. Porter RJ, Gallagher P. Abnormalities of the HPA axis in affective disorders: clinical subtypes and potential treatments. Acta Neuropsych 2006;18:193-209.

4. Goldney RD, Bain MA. Double depression: its morbidity and management in a community setting. Acta Neuropsych 2006;18:210-215. 